YCTP-P-29-99

\title{
Phases of Chiral Gauge Theories
}

\author{
Thomas Appelquist Zhiyong Duant Francesco Sannino \\ Department of Physics, Yale University, New Haven, \\ CT 06520-8120, USA.
}

\begin{abstract}
We discuss the behavior of two non-supersymmetric chiral $S U(N)$ gauge theories, involving fermions in the symmetric and antisymmetric two-index tensor representations respectively. In addition to global anomaly matching, we employ a recently proposed inequality constraint on the number of effective low energy (massless) degrees of freedom of a theory, based on the thermodynamic free energy. Several possible zero temperature phases are consistent with the constraints. A simple picture for the phase structure emerges if these theories choose the phase, consistent with global anomaly matching, that minimizes the massless degree of freedom count defined through the free energy. This idea suggests that confinement with the preservation of the global symmetries through the formation of massless composite fermions is in general not preferred. While our discussion is restricted mainly to bilinear condensate formation, higher dimensional condensates are considered for one case. We conclude by commenting briefly on two related supersymmetric chiral theories.
\end{abstract}

PACS numbers:11.15.-q, 11.30.Rd, 11.15.Ex.

*Electronic address: thomas. appelquist@yale.edu

$\dagger$ Electronic address: zhiyong.duan@yale.edu

$\ddagger$ Electronic address : francesco.sannino@yale.edu 


\section{INTRODUCTION}

Chiral gauge theories, in which at least part of the matter field content is in complex representations of the gauge group, play an important role in efforts to extend the standard model. These include grand unified theories, dynamical breaking of symmetries, and theories of quark and lepton substructure. An important distinction from vector-like theories such as QCD is that since at least some of the chiral symmetries are gauged, mass terms that would explicitly break these chiral symmetries are forbidden in the Lagrangian. Another key feature is that the fermion content is subject to a constraint not present in vectorial gauge theories, the cancellation of gauge and gravitational anomalies.

Chiral theories received much attention in the 1980's [1], focusing on their strong coupling behavior in the infrared. One possibility is confinement with the gauge symmetry as well as global symmetries unbroken, realized by the formation of gauge singlet, massless composite fermions. Another is confinement with intact gauge symmetry but with some of the global symmetries broken spontaneously, leading to the formation of gauge-singlet Goldstone bosons. It is also possible for these theories to exist in the Higgs phase, dynamically breaking their own gauge symmetries [2]. Depending on particle content, they might even remain weakly coupled. This will happen if the theory has an interacting but weak infrared fixed point. The symmetries will then remain unbroken, and the infrared and underlying degrees of freedom will be the same.

Supersymmetric (SUSY) chiral theories have also received considerable attention over the years, since most of the known examples of dynamical supersymmetry breaking involve these kinds of theories [3].

Studies of chiral gauge theories have typically made use of the 't Hooft global anomaly matching conditions [4] along with $1 / N$ expansion, and not-so-reliable most attractive channel (MAC) [5] analysis and instanton computations. Direct approaches using strong coupling lattice methods [6] are still difficult. Another indirect approach developed recently [7] takes the form of an inequality limiting the number of massless degrees of freedom in the infrared description of a field theory to be no larger than the number of ultraviolet degrees of freedom. It was conjectured to apply to all asymptotically free theories whose infrared behavior is also governed by a fixed point, not necessarily free.

The inequality is formulated using finite temperature as a device to probe all energy scales, with the degree-of-freedom count defined using the free energy of the field theory. The zero-temperature theory of interest is characterized using the quantity $f_{I R}$, related to the free energy by 


$$
f_{I R} \equiv-\lim _{T \rightarrow 0} \frac{\mathcal{F}(T)}{T^{4}} \frac{90}{\pi^{2}}
$$

where $T$ is the temperature and $\mathcal{F}$ is the conventionally defined free energy per unit volume. The limit is well defined if the theory has an infrared fixed point. For the special case of an infrared-free theory, $f_{I R}$ is simply the number of massless bosons plus $7 / 4$ times the number of 2-component massless Weyl fermions. The corresponding expression in the large $T$ limit is

$$
f_{U V} \equiv-\lim _{T \rightarrow \infty} \frac{\mathcal{F}(T)}{T^{4}} \frac{90}{\pi^{2}}
$$

This limit will be well defined if the theory has an ultraviolet fixed point. For an asymptotically free theory $f_{U V}$ counts the underlying, ultraviolet degrees of freedom in a similar way.

In terms of these quantities, the conjectured inequality for any asymptotically-free theory is

$$
f_{I R} \leq f_{U V}
$$

This inequality has not been proven, but in Ref. [7] it was shown to agree with all known results and then used to derive new results for several strongly coupled, vector-like gauge theories. It was applied to chiral theories in Ref. [8]. The principal focus there was on the possibility of preserving the global symmetries through the formation of massless composite fermions.

In this paper, we examine further the two non-supersymmetric chiral theories of Ref. [8], both rich in possible phase structure. One is the Bars-Yankielowicz (BY) [9] model involving fermions in the two-index symmetric tensor representation, and the other is a generalized Georgi-Glashow (GGG) model involving fermions in the two-index antisymmetric tensor representation. In each case, in addition to fermions in complex representations, a set of $p$ anti fundamental-fundamental pairs are included and the allowed phases are considered as a function of $p$. Several possible phases emerge, consistent with global anomaly matching and the above inequality.

Graphs for the various $f_{I R}$ 's vs $p$ are plotted for each model. They lead us to the suggestion that each of these theories will choose from among the allowed phases the one that minimizes $f_{I R}$ at each value of $p$. This idea is useful only to distinguish among phases for which the $f_{I R}$ 's are computable, those that are infrared free or governed by a weak infrared fixed point. It may be rephrased in terms of the entropy per unit volume $S(T)$ of the system (the derivative of the pressure per unit volume $P(T)=-\mathcal{F}(T)$ ). Provided only that the 
limit in Eq. (1.1) exists, $S(T)$ near freeze out will be given by $S(T)=\left(2 \pi^{2} / 45\right) T^{3} f_{I R}$ plus higher order terms in the low temperature expansion. Thus the minimum number of degrees of freedom corresponds to the minimum entropy at approach to freeze out, consistent with global anomaly matching. Now of course at any finite $\mathrm{T}$, the preferred phase is chosen from among all the states entering the partition function by minimizing the free energy density, which becomes the energy density at $T=0$. Comparing these quantities for different states when the theory is strongly interacting is, however, generally a strong coupling problem. The conjecture for these theories is the following. Assuming that the candidate phases revealed by anomaly matching are states entering the partition function, the state with the relatively lower energy density is approached at a lower rate proportional to the lower $f_{I R}$. The latter quantity is computable in terms of only the effective low energy theory which is infrared free for the phases being considered here.

We focus almost completely on symmetry breaking patterns corresponding to the formation of bilinear condensates. We suggest that in general, the phase corresponding to confinement with all symmetries unbroken, where all the global anomalies are matched by massless composite fermions, is not preferred. Instead, the global symmetries associated with fermions in real representations break spontaneously via bilinear condensate formation as in QCD. With respect to the fermions in complex representations, however, the formation of bilinear condensates is suggested to be disfavored relative to confinement and the preservation of the global symmetries via massless composite fermion formation.

It is interesting to note that in the real world case of two-flavor QCD (a vector-like theory with all fermions in a real representation) nature prefers to minimize $f_{I R}$. Neglecting the small bare quark masses, global anomaly matching admits two possible low energy phases, broken chiral symmetry through the formation of the bilinear $\langle\bar{\psi} \psi\rangle$ condensate, or unbroken chiral symmetry through the formation of confined massless baryons. Both effective low energy theories are infrared free. The three Goldstone bosons of the former (chosen by nature) lead to $f_{I R}=3$, and the two massless composite Dirac fermions of the latter lead to $f_{I R}=7$.

Bilinear condensate formation is of course not the only possibility in a strongly coupled gauge field theory. We have so far extended our discussion to include general condensate formation for one simple example, the $S U(5)$ Georgi-Glashow model, which has fermions in only complex representations and has only a $U(1)$ global symmetry. This symmetry can be broken via only higher dimensional condensates. Interestingly, this breaking pattern, with confinement and unbroken gauge symmetry, leads to the minimum value of $f_{I R}$. This highlights the important general question of the pattern of symmetry breaking in chiral 
theories when arbitrary condensate formation is considered. Higher dimensional condensates might play an important role, for example, in the dynamical breaking of symmetries in extensions of the standard model [10].

The Bars-Yankielowicz model is discussed in Section II and the generalized GeorgiGlashow model is discussed in Section III. In Section IV, we briefly describe two supersymmetric chiral theories: the supersymmetric version of the $S U(5)$ Georgi-Glashow model and the closely related $3-2$ model. In Section $\nabla$ we summarize and conclude.

\section{THE BARS YANKIELOWICZ (BY) MODEL}

This model is based on the single gauge group $S U(N \geq 3)$ and includes fermions transforming as a symmetric tensor representation, $S=\psi_{L}^{\{a b\}}, a, b=1, \cdots, N ; N+4+p$ conjugate fundamental representations: $\bar{F}_{a, i}=\psi_{a, i L}^{c}$, where $i=1, \cdots, N+4+p$; and $p$ fundamental representations, $F^{a, i}=\psi_{L}^{a, i}, i=1, \cdots, p$. The $p=0$ theory is the basic chiral theory, free of gauge anomalies by virtue of cancellation between the antisymmetric tensor and the $N+4$ conjugate fundamentals. The additional $p$ pairs of fundamentals and conjugate fundamentals, in a real representation of the gauge group, lead to no gauge anomalies.

The global symmetry group is

$$
G_{f}=S U(N+4+p) \times S U(p) \times U_{1}(1) \times U_{2}(1)
$$

Two $U(1)$ 's are the linear combination of the original $U(1)$ 's generated by $S \rightarrow e^{i \theta_{S} S}$, $\bar{F} \rightarrow e^{i \theta_{\bar{F}}} \bar{F}$ and $F \rightarrow e^{i \theta_{F}} F$ that are left invariant by instantons, namely that for which $\sum_{j} N_{R_{j}} T\left(R_{j}\right) Q_{R_{j}}=0$, where $Q_{R_{j}}$ is the $U(1)$ charge of $R_{j}$ and $N_{R_{j}}$ denotes the number of copies of $R_{j}$.

Thus the fermionic content of the theory is

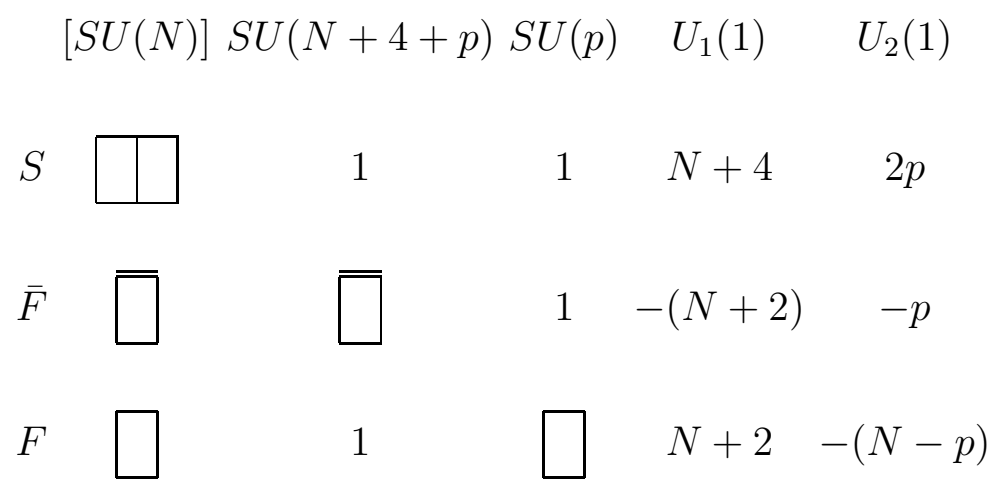

where the first $S U(N)$ is the gauge group, indicated by the square brackets.

For all the models considered in this paper, the beta function is generically written as 


$$
\beta=\mu \frac{d \alpha}{d \mu}=-\beta_{1}\left(\frac{\alpha^{2}}{2 \pi}\right)-\beta_{2}\left(\frac{\alpha^{3}}{4 \pi^{2}}\right)+O\left(\alpha^{4}\right),
$$

where the terms of order $\alpha^{4}$ and higher are scheme-dependent. For the present model, we have $\beta_{1}=3 N-2-(2 / 3) p$ and $\beta_{2}=(1 / 4)\left\{13 N^{2}-30 N+1+12 / N-2 p((13 / 3) N-1 / N)\right\}$. Thus the theory is asymptotically free for

$$
p<(9 / 2) N-3
$$

We shall restrict $p$ so that this condition is satisfied.

Because of asymptotic freedom, the thermodynamic free-energy may be computed in the $T \rightarrow \infty$ limit. An enumeration of the degrees of freedom leads to

$$
f_{U V}=2\left(N^{2}-1\right)+\frac{7}{4}\left[\frac{N(N+1)}{2}+(N+4) N+2 p N\right]
$$

The infrared realization of this theory will vary depending on the number $p$ of conjugate fundamental-fundamental pairs. We begin by discussing the $p=0$ theory and then map out the phase structure as function of $p$.

\section{A. The $p=0$ Case}

For $p=0$, the fermions are in complex representations of the $S U(N)$ gauge group and the global symmetry group is $G_{f}=S U(N+4) \times U_{1}(1)$. The theory is strongly coupled at low energies, so it is expected either to confine or to break some of the symmetries, consistent with global anomaly matching.

The possibility that the $p=0$ theory confines with the full global symmetry group $G_{f}$ unbroken has been considered previously in the literature [8,11]. All the global anomalies of the underlying theory may be matched at low energies providing that the massless spectrum is composed of gauge singlet composite fermions transforming according to the antisymmetric second-rank tensor representation of $S U(N+4)$. They are described by the composite operators $\bar{F}_{[i} S \bar{F}_{j]}$ and have charge $-N$ under the $U_{1}(1)$ global symmetry.

With only these massless composites in the low energy spectrum, there are no dimensionfour interactions, so the composites are noninteracting in the infrared. Therefore the thermodynamic free energy may be computed in the limit $T \rightarrow 0$. Enumerating the degrees of freedom gives

$$
f_{I R}^{s y m}(p=0)=\frac{7}{4} \frac{(N+4)(N+3)}{2},
$$


where the superscript indicates that the full global symmetry is intact. Clearly $f_{I R}^{s y m}(p=$ $0)<f_{U V}(p=0)$, satisfying the inequality of Eq. (1.3) 8].

While the formation of confined massless composite fermions and the preservation of $G_{f}$ is consistent with anomaly matching and the thermal inequality, the same can be seen to be true of broken symmetry channels. We consider first the Higgs phase corresponding to the maximally attractive channel. It is [5]

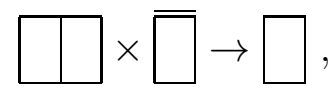

leading to the formation of the $S \bar{F}$ condensate

$$
\varepsilon^{\gamma \delta} S_{\gamma}^{a i} \bar{F}_{\{a, i\}, \delta}
$$

where $\gamma, \delta=1,2$ are spin indices and $a, i=1, \cdots, N$ are gauge and flavor indices. This condensate breaks $U_{1}(1)$ and all the gauge symmetries, and it breaks $S U(N+4)$ to $S U(4)$. But the $S U(N)$ subgroup of $S U(N+4)$ combines with the gauge group, leading to a new global symmetry $S U^{\prime}(N)$. For this group, $\bar{F}_{a, i \leq N}$ is reducible, to the symmetric $\bar{F}^{S}=\bar{F}_{\{a, i\}}$ and the anti-symmetric $\bar{F}^{A}=\bar{F}_{[a, i]}$ representations.

The broken $S U(N+4)$ generator

$$
Q_{(N+4)}=\left(\begin{array}{ll|lll}
4 & & & & \\
& \ddots & & & \\
& 4 & & \\
\hline & -N & & \\
& & & \ddots & \\
& & & & -N
\end{array}\right),
$$

combines with $Q_{1}$ giving a residual global symmetry $U_{1}^{\prime}(1)=\frac{1}{N+4}\left(2 Q_{1}-Q_{(N+4)}\right)$. The breakdown pattern thus is

$$
[S U(N)] \times S U(N+4) \times U_{1}(1) \rightarrow S U^{\prime}(N) \times S U(4) \times U_{1}^{\prime}(1)
$$

The gauge bosons have become massive as have some fermions. The fermionic spectrum, with respect to the residual global symmetry is 


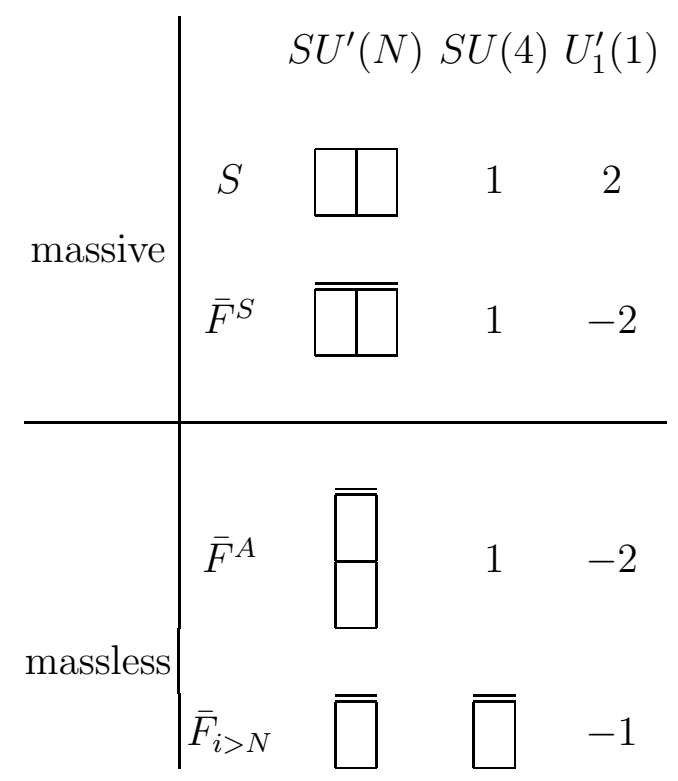

This breaking pattern gives $N^{2}+8 N$ Goldstone bosons, $N^{2}-1$ of which are eaten by the gauge bosons. So only $8 N+1$ remain as part of the massless spectrum along with the massless fermions. The global anomalies are again matched by this spectrum. Those associated with the unbroken group $S U^{\prime}(N) \times S U(4) \times U_{1}^{\prime}(1)$ are matched by the massless fermions, while those associated with the broken global generators are matched by the Goldstone bosons. Since the Goldstone bosons do not couple singly to the massless fermions (no dimension-four operators), the effective zero-mass theory is free at low energies.

It follows that the thermodynamic free energy may be computed at $T \rightarrow 0$ by counting the degrees of freedom. The result is

$$
f_{I R}^{\text {Higgs }}(p=0)=(8 N+1)+\frac{7}{4}\left[\frac{1}{2} N(N-1)+4 N\right],
$$

where the superscript indicates that the gauge symmetry is (partially) broken. Just as in the case of the symmetric phase, the inequality Eq. (1.3) is satisfied: $f_{I R}^{H i g g s}(p=0)<f_{U V}(p=0)$.

As an aside, we note that according to the idea of complementarity this low energy phase may be thought of as having arisen from confining gauge forces rather than the Higgs mechanism [12,13]. Confinement then would partially break the global symmetry to the above group forming the necessary Goldstone bosons. It would also produce gauge singlet massless composite fermions to replace precisely the massless elementary fermions in the above table.

We have identified two possible phases of this theory consistent with global anomaly matching and the inequality Eq. (1.3). One confines and breaks no symmetries. The other breaks the chiral symmetry according to Eq. (2.8). For any finite value of $N, f_{I R}^{s y m}(p=0)<$ $f_{I R}^{\text {Higgs }}(p=0)$. The symmetric phase is thus favored if the number of degrees of freedom, or 
the entropy of the system near freeze-out, is minimized. In the limit $N \rightarrow \infty$, the Goldstone bosons do not contribute to leading order, and $f_{I R}^{\text {sym }}(p=0) \rightarrow f_{I R}^{\text {Higgs }}(p=0)$. We return to a discussion of the infinite $N$ limit after describing the general $(p>0)$ model.

What about other symmetry breaking phases of the $p=0$ theory corresponding to bilinear condensate formation? In addition to $S \bar{F}$ condensates, there are also $S S$ and $\bar{F} \bar{F}$ possibilities. Several of these correspond to attractive channels, although not maximally attractive, due to gluon exchange. We have considered all of them for the case $N=3$, and have shown that the effective low energy theory is infrared free and that the number of low energy degrees of freedom is larger than the symmetric phase. Whether this is true for symmetry breaking patterns corresponding to bilinear condensate formation for general $N$ remains to be seen. Higher dimensional condensate formation is yet to be studied for any of these choices.

\section{B. The General Case}

We next consider the full range of $p$ allowed by asymptotic freedom: $0<p<(9 / 2) N-3$. For $p$ near $(9 / 2) N-3$, an infrared stable fixed point exists, determined by the first two terms in the $\beta$ function. This can be arranged by taking both $N$ and $p$ to infinity with the difference $(9 / 2) N-p$ fixed, or at finite $N$ by continuing to nonintegral $p$. The infrared coupling is then weak and the theory neither confines nor breaks symmetries. The fixed point leads to an approximate, long-range conformal symmetry. As $p$ is reduced, the screening of the long range force decreases, the coupling increases, and confinement and/or symmetry breaking set in. We consider three strong-coupling possibilities, each consistent with global anomaly matching.

\section{Confinement with no symmetry Breaking}

It was observed by Bars and Yankielowicz [9] that confinement without chiral symmetry breaking is consistent with global anomaly matching provided that the spectrum of the theory consists of massless composite fermions transforming under the global symmetry group as follows: 


$$
\begin{aligned}
& {[S U(N)] S U(N+4+p) S U(p) U_{1}(1) U_{2}(1)} \\
& \begin{array}{llllll}
\bar{F} S \bar{F} & 1 & \square & 1 & -N & 0
\end{array}
\end{aligned}
$$

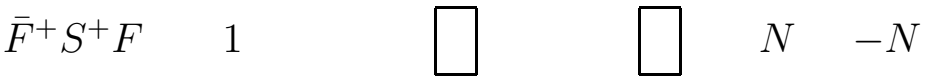

$$
\begin{aligned}
& \begin{array}{llllll}
F^{+} S F^{+} & 1 & 1 & \square \square & -N & 2 N
\end{array}
\end{aligned}
$$

The effective low-energy theory is free. In Ref. [8], the thermodynamic free energy for this phase was computed, giving

$$
f_{I R}^{s y m}=\frac{7}{4}\left[\frac{1}{2}(N+4+p)(N+3+p)+p(N+4+p)+\frac{1}{2} p(p+1)\right] .
$$

The inequality $f_{I R}^{s y m}<f_{U V}$ was then invoked to argue that this phase is possible only if $p$ is less than a certain value (less than the asymptotic freedom bound). For large $N$, the condition is $p<(15 / 14)^{1 / 2} N$.

\section{Chiral symmetry breaking}

Since this theory is vector-like with respect to the $p F-\bar{F}$ pairs, it may be anticipated that these pairs condense according to

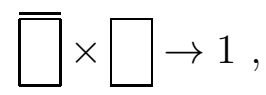

leading to a partial breaking of the chiral symmetries. The gauge-singlet bilinear condensate (fermion mass) is of the form

$$
\varepsilon^{\gamma \delta} F_{\gamma}^{a, i} \bar{F}_{a, N+4+i, \delta}
$$

where $i=1, \ldots, p$.

This leads to the symmetry breaking pattern $S U(N+4+p) \times S U(p) \times U_{1}(1) \times U_{2}(1) \rightarrow$ $S U(N+4) \times S U_{V}(p) \times U_{1}^{\prime}(1) \times U_{2}^{\prime}(1)$, producing $2 p N+p^{2}+8 p$ gauge singlet Goldstone bosons. The $U^{\prime}(1)^{\prime} s$ are combinations of the $U(1)^{\prime} s$ and the broken generator of $S U(N+4+p)$ 


$$
Q_{(N+4+p)}=\left(\begin{array}{ll|lll}
-p & & & & \\
& \ddots & & & \\
& & & & \\
& & & & \\
\hline & & N+4 & \\
& & & \ddots & \\
& & & N+4
\end{array}\right) .
$$

At this stage, the remaining massless theory is the $p=0$ theory described above, together with the $2 p N+p^{2}+8 p$ gauge-singlet Goldstone bosons. Since the Goldstone bosons are associated with the broken symmetry, there will be no dimension-four interactions between them and the $p=0$ theory. This theory may therefore be analyzed at low energies by itself, leading to the possible phases described above. Two possible phases of the $p=0$ theory were discussed in detail. One corresponds to confinement and massless composite fermion formation with no chiral symmetry breaking. For the general theory, this corresponds to

- Partial chiral symmetry breaking but no gauge symmetry breaking. The vector-like $p$ pairs of $F$ and $\bar{F}$ condense, and others form composite fermions.

The massless spectrum consists of the $2 p N+p^{2}+8 p$ Goldstone bosons together with the $(N+4)(N+3) / 2$ composite fermions of the $p=0$ sector. All are confined. The final global symmetry is $S U(N+4) \times S U_{V}(p) \times U_{1}^{\prime}(1) \times U_{2}^{\prime}(1)$. Global anomalies are matched partially by the massless composites and partially by the Goldstone bosons. Since both theories are infrared free, the free energy may be computed in the $T \rightarrow 0$ limit to give

$$
f_{I R}^{b r k+s y m}=\left(2 p N+p^{2}+8 p\right)+\frac{7}{4}\left[\frac{1}{2}(N+4)(N+3)\right] .
$$

The inequality Eq. (1.3) thus allows this phase for $p$ less than a certain value below the asymptotic freedom bound but above the value at which the symmetric phase becomes possible. For large $\mathrm{N}$, the limit is $p / N$ less than $\simeq 2.83$.

The other phase of the $p=0$ theory considered above, corresponds to the MAC for symmetry breaking and the Higgsing of the gauge group with a further breaking of the chiral symmetry. For the general theory $(p>0)$, it leads to

- Further chiral symmetry breaking and gauge symmetry breaking.

The final global symmetry is $S U^{\prime}(N) \times S U(4) \times S U_{V}(p) \times U_{1}^{\prime}(1) \times U_{2}^{\prime}(1)$. The massless spectrum consists of the $2 p N+p^{2}+8 p$ Goldstone bosons associated with the $p F-\bar{F}$ pairs, 
together with the $8 N+1$ Goldstone bosons and $N(N+1) / 2+4 N$ massless elementary fermions of the $p=0$ sector. Global anomalies are matched partially by Goldstone bosons and partially by the remaining massless fermions. The effective low energy theories are infrared free, and we have

$$
\begin{aligned}
f_{I R}^{b r k+H i g g s}= & \left(2 p N+p^{2}+8 p\right)+(8 N+1) \\
& +\frac{7}{4}\left[\frac{1}{2} N(N-1)+4 N\right] .
\end{aligned}
$$

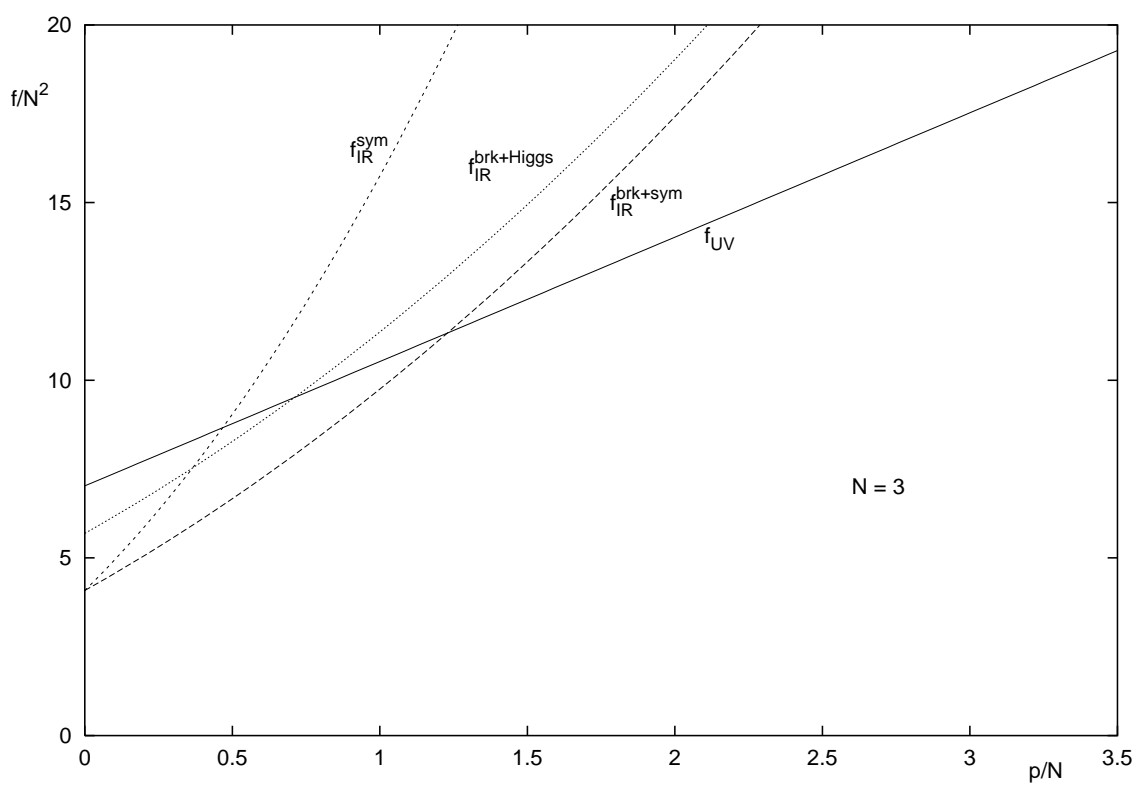

FIG. 1. BY Model: Degree of freedom count $f$ (normalized to $N^{2}$ ) for different phases as function of the number $p$ of $F-\bar{F}$ pairs for the choice $N=3 . f_{I R}^{s y m}$ indicates confinement with intact chiral global symmetry while $f_{I R}^{b r k+s y m}$ indicates confinement with partial chiral symmetry breaking. $f_{I R}^{b r k+H i g g s}$ indicates partial chiral symmetry breaking with gauge symmetry breaking. $f_{U V}$ counts the underlying degrees of freedom. As $N$ increases, the $f_{I R}^{b r k+s y m}$ and $f_{I R}^{b r k+H i g g s}$ curves approach each other.

Three possible phases of the general Bars-Yankielowicz model have now been identified. In Fig. 1, we summarize the computation of $f_{I R} / N^{2}$ for each phase and compare with $f_{U V}$ for the choice $N=3$. Other choices are qualitatively the same. Each phase satisfies the inequality Eq. (1.3) for $p / N$ small enough. As $p$ is reduced, the first phase allowed by the inequality corresponds to confinement with condensation of the $p$ fermions in the real representation of the gauge group and the breaking of the associated chiral symmetry, along 
with unbroken chiral symmetry and massless composite fermion formation in the $p=0$ sector. The degree of freedom count is denoted by $f_{I R}^{b r k+s y m}$.

The two other phases are also allowed by the inequality as $p$ is reduced further. But for any finite value of $N$ and for any value of $p>0$, the curve for $f_{I R}^{b r k+s y m}$ is the lowest of the $f_{I R}$ curves. Thus the lowest infrared degree-of-freedom count corresponds to a complete breaking of the chiral symmetry associated with the $p F-\bar{F}$ pairs (the vector- like part of the theory with the fermions in a real representation of the gauge group), and no breaking of the chiral symmetry associated with the $p=0$ sector (the part of the theory with the fermions in complex representations).

It is instructive to examine this model in the infinite $N$ limit. If the limit is taken with $p / N$ fixed, the curves for $f_{I R}^{b r k+s y m} / N^{2}$ and $f_{I R}^{b r k+H i g g s} / N^{2}$ become degenerate for all values of $p / N$, and are below the curve for $f_{I R}^{s y m}$. If the limit $N \rightarrow \infty$ is taken with $p$ fixed, all the curves become degenerate, and the phases are not distinguished by the number of degrees of freedom. The authors of Ref. [1] analyzed the model in the $N \rightarrow \infty$ limit with confinement assumed and noted that the $U_{1}(1)$ symmetry cannot break because no appropriate order parameter can form in this limit. This is consistent with the above discussion since each of the phases preserves the $U_{1}(1)$ for any $N$.

As with the $p=0$ theory, there are other possible symmetry breaking phases corresponding to bilinear condensate formation. Some of these are attractive channels, although not maximally attractive, due to gluon exchange. We have considered several possibilities. Each leads to an effective low energy theory that is infrared free, and each gives a larger value of $f_{I R}$ than the phase corresponding to the lowest curve in Fig. 1: complete breaking of the chiral symmetry associated with $p$ additional $F-\bar{F}$ pairs and no breaking of the chiral symmetry associated with the sector of the theory with the fermions in complex representations.

Symmetry breaking by higher dimensional condensate formation is yet to be considered, and we have nothing to say about possible strongly coupled infrared phases such as a strongly coupled nonabelian Coulomb phase.

\section{THE GENERALIZED GEORGI-GLASHOW (GGG) MODEL}

This model is similar to the BY model just considered. It is an $S U(N \geq 5)$ gauge theory, but with fermions in the anti-symmetric, rather than symmetric, tensor representation. The complete fermion content is $A=\psi_{L}^{[a b]}, a, b=1, \cdots, N$; an additional $N-4+p$ fermions in the conjugate fundamental representations: $\bar{F}_{a, i}=\psi_{a, i L}^{c}, i=1, \cdots, N-4+p$; and $p$ fermions in the fundamental representations, $F^{a, i}=\psi_{L}^{a, i}, i=1, \cdots, p$. 
The global symmetry is

$$
G_{f}=S U(N-4+p) \times S U(p) \times U_{1}(1) \times U_{2}(1)
$$

where the two $U(1)$ 's are anomaly free. With respect to this symmetry, the fermion content is

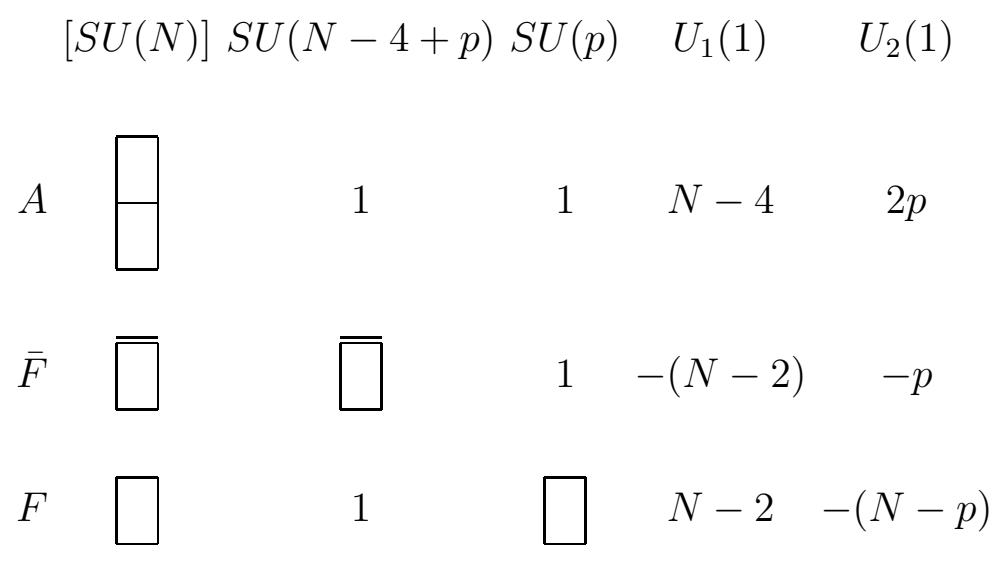

For the $\beta$-function, we have $\beta_{1}=3 N+2-(2 / 3) p$ and $\beta_{2}=(1 / 4)\left\{13 N^{2}+30 N+1+\right.$ $12 / N-2 p((13 / 3) N-1 / N)\}$. Thus the theory is asymptotically free if

$$
p<(9 / 2) N+3
$$

We restrict $p$ so that this condition is satisfied. Because of asymptotic freedom, the thermodynamic free-energy may be computed in the $T \rightarrow \infty$ limit. We have

$$
f_{U V}=2\left(N^{2}-1\right)+\frac{7}{4}\left[\frac{N(N-1)}{2}+(N-4) N+2 p N\right]
$$

As with the BY model, we first discuss the $p=0$ theory and then consider the general case.

\section{A. The $p=0$ Case}

The global symmetry group is $G_{f}=S U(N-4) \times U_{1}(1)$. The theory is strongly coupled at low energies, so it is expected either to confine or to break some of the symmetries, consistent with global anomaly matching [4].

In the case of complete confinement and unbroken symmetry, to satisfy global anomaly matching the massless spectrum consists of gauge singlet composite fermions $\bar{F}_{\{i} A \bar{F}_{j\}}$ transforming according to the symmetric second-rank tensor representation of $S U(N-4)$ with charge $-N$ under the $U_{1}(1)$ global symmetry [9]. The composites are noninteracting in the 
infrared. Therefore the thermodynamic free energy may be computed in the limit $T \rightarrow 0$. Enumerating the degrees of freedom gives

$$
f_{I R}^{s y m}(p=0)=\frac{7}{4} \frac{(N-4)(N-3)}{2} .
$$

Clearly $f_{I R}^{\text {sym }}(p=0)<f_{U V}(p=0)$, satisfying the inequality Eq. (1.3).

We next consider symmetry breaking due to bilinear condensate formation by first examining the maximally attractive channel [5]:

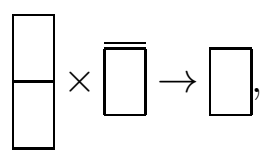

leading to the formation of the $A \bar{F}$ condensate

$$
\varepsilon^{\gamma \delta} A_{\gamma}^{a i} \bar{F}_{a, i, \delta}
$$

where $\gamma, \delta=1,2$ are spin indices, $a=1, \cdots, N$, is a gauge index and $i=1, \cdots, N-4$ is a flavor index. This condensate breaks the $U_{1}(1)$ symmetry and breaks the gauge symmetry $S U(N)$ to $S U(4)$. The broken gauge subgroup $S U(N-4)$ combines with the flavor group, leading to a new global symmetry $S U^{\prime}(N-4)$, while the broken gauge $S U(N)$ generator

$$
Q_{(N)}=\left(\begin{array}{ll|lll}
4 & & & & \\
& \ddots & & & \\
& 4 & & & \\
& & 4-N & & \\
& & & \ddots & \\
& & & & 4-N
\end{array}\right),
$$

combines with $U_{1}(1)$ to form a residual global symmetry $U^{\prime}(1)$. The remaining symmetry is thus $[S U(4)] \times S U^{\prime}(N-4) \times U_{1}^{\prime}(1)$. All Goldstone bosons are eaten by gauge bosons.

We have

$$
\bar{F}_{a, i}=\left(\frac{\bar{F}_{j, i} \rightarrow \bar{F}_{[j, i]}+\bar{F}_{\{j, i\}}}{\bar{F}_{c, i}}\right)
$$

and

$$
A^{a b}=\left(\begin{array}{l|l}
A^{i j} & A^{i c} \\
\hline & A^{c d}
\end{array}\right),
$$


where $a, b=1, \cdots, N, i, j=1, \cdots, N-4$, and $c, d=N-3, \cdots, N$. The $A \bar{F}$ condensate pairs $\bar{F}_{[j, i]}$ with $A^{i j}$ and $\bar{F}_{c, i}$ with $A^{i c}$. This leaves only $A^{c d}$, which is neutral under $U^{\prime}(1)$, as the fermion content of the $S U(4)$ gauge theory.

This $S U(4)$ theory is also strongly coupled in the infrared and we expect it to confine. The most attractive channel for condensate formation, for example, is

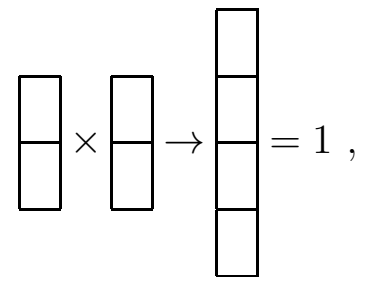

leading to the bilinear condensate

$$
\varepsilon^{\gamma \delta} A_{\gamma}^{a b} A_{\delta}^{c d} \varepsilon_{1 \cdots(N-4) a b c d}
$$

a singlet under the gauge group. Thus, in the infrared, the only massless fermions are the $\bar{F}_{\{j, i\}}^{\prime} s$ in the symmetric two-index tensor representation of $S U^{\prime}(N-4)$. Interestingly, the massless fermion content and the low energy global symmetry are precisely the same for the symmetric and Higgs phases. Therefore,

$$
f_{I R}^{h i g g s}(p=0)=f_{I R}^{s y m}(p=0)=\frac{7}{4}\left[\frac{1}{2}(N-4)(N-3)\right] .
$$

The fermions are composite in the first case and elementary in the second. This is another example of the complementarity idea [13]. While the two phases are not distinguished by the low energy considerations used here, they are different phases. However, other ideas involving energies on the order of the confinement and/or breaking scales will have to be employed to distinguish them.

A general study of the phases of chiral gauge theories should include higher dimensional as well as bilinear condensate formation. We have done this for one case, the $p=0 S U(N=5)$ model, which possesses only a $U(1)$ global symmetry. Among the various phases that may be considered is one that confines but breaks the global $U(1)$. This corresponds to the formation of gauge invariant higher dimensional condensates, for example of the type $(\bar{F} A \bar{F})^{2}$. There is no bilinear condensate for this breaking pattern. Global anomaly matching is satisfied by the appearance of a single massless Goldstone boson and no other massless degrees of freedom. This phase clearly minimizes the degree of freedom count (the entropy near freezeout), among the phases described by infrared free effective theories. The unbroken phase, by contrast, must include a massless composite fermion for anomaly matching, and therefore gives a larger $f_{I R}$. This suggests that higher dimensional condensate formation may indeed 
be preferred in this model. It will be interesting to study this possibility in more detail and to see whether higher dimensional condensate formation plays an important role in the larger class of chiral theories considered here and in other theories.

\section{B. The General Case}

The full range of $p$ allowed by asymptotic freedom may be considered just as it was for the BY model. For $p$ near $(9 / 2) N+3$, an infrared stable fixed point exists, determined by the first two terms in the $\beta$ function. The infrared coupling is then weak and the theory neither confines nor breaks symmetries. As $p$ decreases, the coupling strengthens, and confinement and/or symmetry breaking set in. We consider two possibilities consistent with global anomaly matching.

\section{Confinement with no symmetry breaking}

It is known [9] that confinement without chiral symmetry breaking is consistent with global anomaly matching provided that the spectrum of the theory consists of gauge singlet massless composite fermions transforming under the global symmetry group as follows:

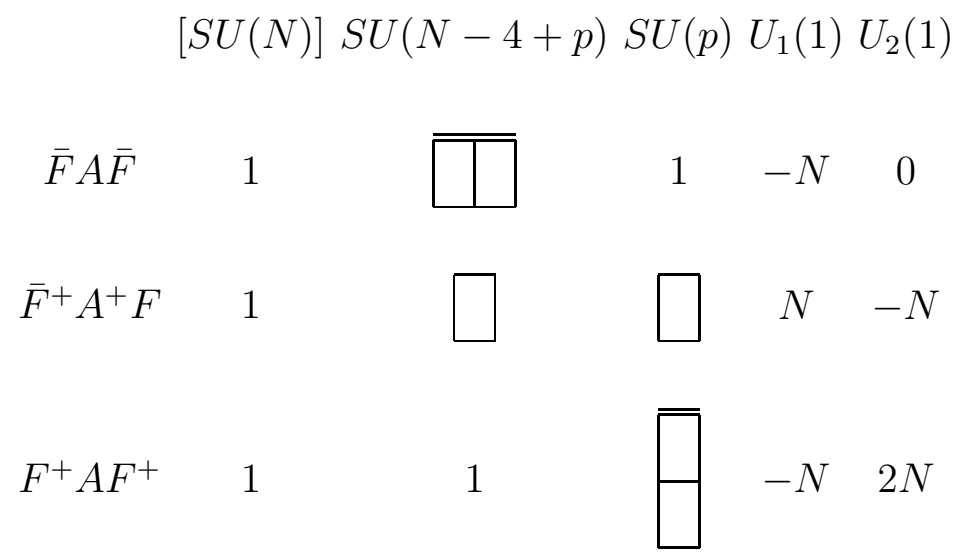

The effective low energy is free. Thus the thermodynamic free energy may be computed in the limit $T \rightarrow 0$ to give

$$
f_{I R}^{\text {sym }}=\frac{7}{4}\left[\frac{1}{2}(N-4+p)(N-3+p)+p(N-4+p)+\frac{1}{2} p(p-1)\right] .
$$

The inequality Eq. (1.3) allows this phase when $p / N$ is less than $\simeq 2.83$, for large $N$. 


\section{Chiral symmetry breaking}

As in the BY model it may be expected that the fermions in a real representation of the gauge group (the $p F-\bar{F}$ pairs) will condense in the pattern

$$
\square \times \square \rightarrow 1
$$

The gauge-singlet bilinear condensate (fermion mass) is of the form

$$
\varepsilon^{\gamma \delta} F_{\gamma}^{a, i} \bar{F}_{a, N-4+i, \delta}
$$

where $i=1, \ldots, p$, leading to the symmetry breaking pattern

$$
\begin{aligned}
& S U(N-4+p) \times S U(p) \times U_{1}(1) \times U_{2}(1) \\
& \rightarrow S U(N-4) \times S U_{V}(p) \times U_{1}^{\prime}(1) \times U_{2}^{\prime}(1)
\end{aligned}
$$

and producing $2 p N+p^{2}-8 p$ gauge singlet Goldstone bosons.

The $U^{\prime}(1)^{\prime} s$ are combinations of the $U(1)^{\prime} s$ and the broken generator of $S U(N-4+p)$

$$
Q_{(N-4+p)}=\left(\begin{array}{ll|lll}
-p & & & & \\
& \ddots & & & \\
& & & & \\
& & N-4 & \\
& & & \ddots & \\
& & & N-4
\end{array}\right) .
$$

The remaining massless theory is the $p=0$ theory described above, together with the $2 p N+p^{2}-8 p$ gauge-singlet Goldstone bosons. Since the Goldstone bosons are associated with the broken symmetry, there will be no dimension-four (Yukawa) interactions between them and the $p=0$ fields. The $p=0$ theory may therefore be analyzed by itself, leading to the possible phases described above. Two phases were considered, one symmetric and the other broken by the maximally attractive bilinear condensate, and they were seen to lead to identical low energy theories.

Thus, in either case, the degree-of-freedom count for the general theory, corresponding to the breaking of the chiral symmetry associated with the $p F-\bar{F}$ pairs, gives

$$
f_{I R}^{b r k}=\left(2 p N+p^{2}-8 p\right)+\frac{7}{4}\left[\frac{1}{2}(N-4)(N-3)\right] .
$$

To summarize, two possible phases of the general GGG model have been considered. In Fig. 2, we plot the two computations of $f_{I R} / N^{2}$ for the choice $N=6$ and compare them 


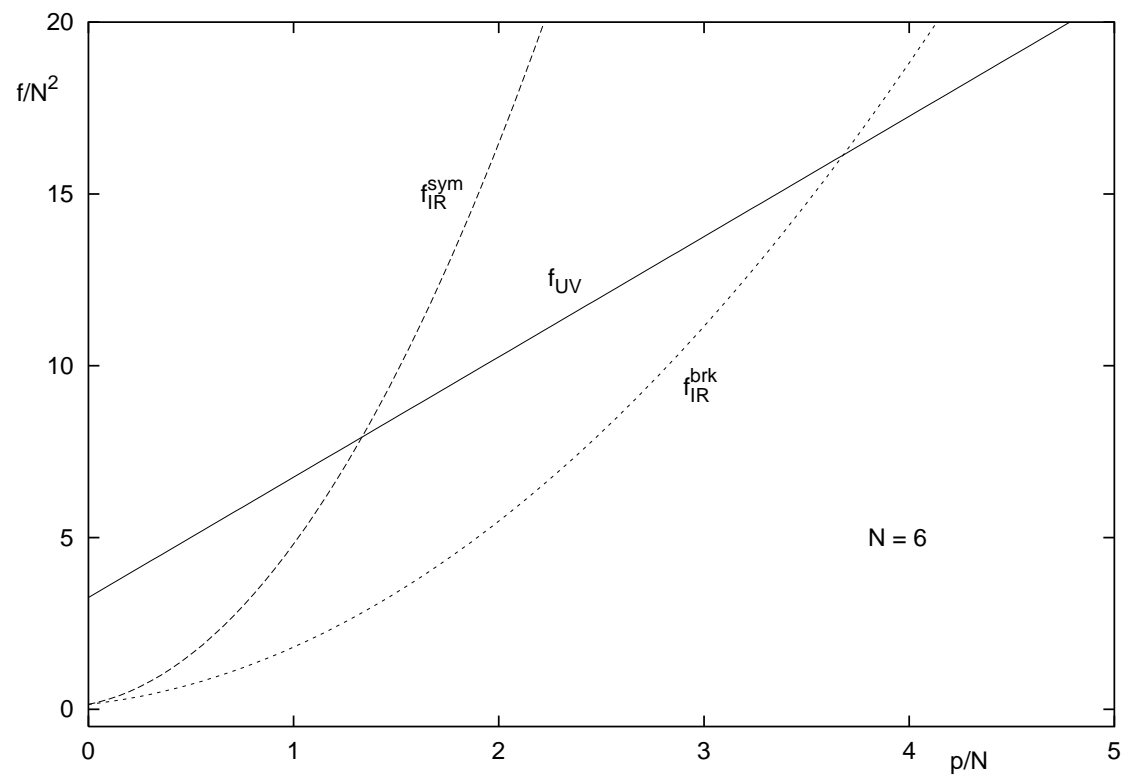

FIG. 2. GGG Model: Degree of freedom count $f$ (normalized to $N^{2}$ ) for different phases as function of the number $p$ of $F-\bar{F}$ pairs for the choice $N=6$.

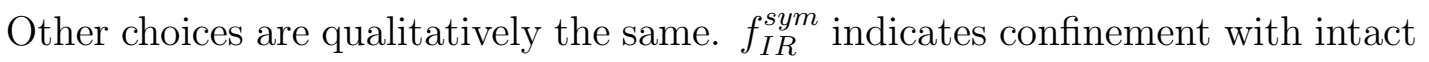
chiral global group while $f_{I R}^{b r k}$ indicates either confinement or gauge symmetry breaking, with partial chiral symmetry breaking. $f_{U V}$ counts the underlying degrees of freedom.

with $f_{U V}$. Each phase satisfies the inequality Eq. (1.3) for $p$ below some value. As $p$ is reduced, the first phase allowed by the inequality corresponds to partial chiral symmetry breaking. For any $p, f_{I R}^{b r k}$ is the lower of the $f_{I R}$ curves. Thus the lower infrared degree-offreedom count corresponds to a complete breaking of the chiral symmetry associated with $p$ additional $F-\bar{F}$ pairs (the vector like part of the theory) and no breaking of the chiral symmetry associated with the fermions in a complex representation of the gauge group. Whether the latter behavior is due to confinement or the Higgsing of the gauge group has not been determined. These conclusions remain valid in the infinite $N$ limit with $p / N$ fixed. If the limit $N \rightarrow \infty$, is taken with $p$ fixed, the two curves become degenerate.

As we have already noted, for the $p=0 S U(5)$ theory there is a still lower degree of freedom count when higher dimensional condensates are considered. This will therefore also be true of the general- $p$ case for $S U(5)$. This even lower count corresponds to complete confinement along with breaking of the chiral symmetry associated with the $p F-\bar{F}$ pairs and breaking of the remaining global $U(1)$ symmetry. It will be interesting to see whether a preference for this phase can be confirmed by a dynamical study of this model and whether 
similar higher dimensional condensate formation is favored in a more general class of models.

\section{TWO CHIRAL SUSY MODELS}

Although this paper is devoted principally to non-SUSY chiral models, we briefly describe two chiral SUSY models: the supersymmetric generalization of the one generation $S U(5)$ Georgi-Glashow model [14] and the related $(3-2)$ model (see [3] for a review of this model and relevant references).

The $S U(5)$ model contains a single antisymmetric tensor chiral superfield $A$ and an antifundamental chiral superfield $\bar{F}$. The vector superfield $W_{\alpha}$ includes the standard vector boson and the associated gluino in the adjoint representation of $S U(5)$. The global symmetry is the anomaly-free $U_{R}(1) \times U_{A}(1)$, and the charge assignments are:

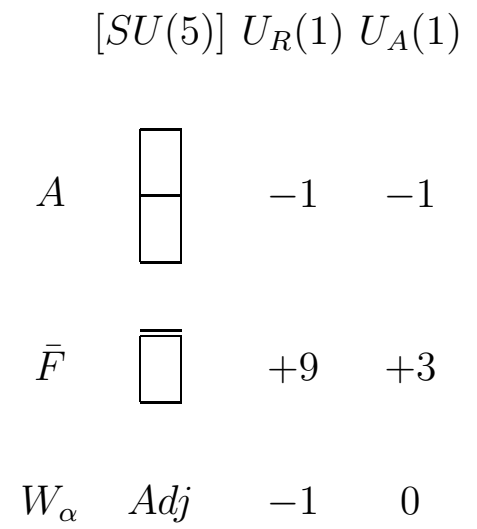

A special feature of this model is that the classical vacuum is unique. The absence of flat directions is due to the fact that there exists no holomorphic gauge invariant polynomial constructed out of the supersymmetric fields. This feature guarantees that when comparing phases through their degree-of-freedom count, we know that we are considering a single underlying theory. By contrast, in SUSY gauge theories with flat directions, non-zero condensates associated with the breaking of global symmetries correspond to different points in moduli space and therefore to different theories.

This model was studied long ago [14] and various possible phases were seen to be consistent with global anomaly matching. One preserves supersymmetry along with the global symmetries. This requires composite massless fermions to saturate the global anomalies. It was shown that there are several, rather complicated, solutions, with at least five Weyl fermions (which for supersymmetry to hold must be cast in five chiral superfields). The charge assignments for one of them is 14]: 


$$
(-5,-26),(5,20),(5,24),(0,-1),(0,9)
$$

where the first entry is the $U_{A}(1)$ charge and the second is the $U_{R}(1)$ charge of each chiral superfield.

Other possibilities are that SUSY breaks with the global symmetries unbroken or that one or both of the global symmetries together with supersymmetry break spontaneously. It is expected [14] that in a supersymmetric theory without classical flat directions, the spontaneous breaking of global symmetries also signals spontaneous supersymmetry breaking. In these cases, the only massless fields will be the Goldstone boson(s) associated with the broken global symmetries and/or some massless fermions transforming under the unbroken chiral symmetries, together with the Goldstone Weyl fermion associated with the spontaneous supersymmetry breaking.

In Reference [14] it was suggested on esthetic grounds that the supersymmetric solution seems less plausible. Additional arguments that supersymmetry is broken are based on investigating correlators in an instanton background [15]. However a firm solution to this question is not yet available.

Since all the above phases are non interacting in the infrared we may reliably compute $f_{I R}$ and note that the phase that minimizes the degree-of-freedom count is the one that breaks supersymmetry and both of the global symmetries. This phase consists of two $U(1)$ Goldstone bosons and a single Weyl Goldstino associated with the breaking of SUSY. Thus $f_{I R}=15 / 4$. SUSY preserving phases and those that leave one or both of the $U(1)^{\prime} s$ unbroken lead to more degrees of freedom. It will be interesting to see whether further dynamical studies confirm that the maximally broken phase is indeed preferred

This phase is similar to the minimal- $f_{I R}$ phase in the nonsupersymmetric $S U(5)$ model in that both correspond to higher dimensional condensate formation. In the SUSY case, one can construct two independent order parameters. The one for $U_{R}(1)$ is the gluino condensate (scalar component of the chiral superfield $W^{\alpha} W_{\alpha}$ ) while the one for $U_{A}(1)$ can be taken to be the scalar component of the chiral superfield $\bar{F}_{a} \bar{F}_{b} A^{a c}\left(W^{\alpha} W_{\alpha}\right)_{c}^{b}$.

Finally we comment on a well known and related chiral model for dynamical supersymmetry breaking: the $(3-2)$ model. Unlike the models considered so far, this model involves multiple couplings, i.e. two gauge couplings and a Yukawa one. Without the Yukawa interaction the theory posses a run-away vacuum. The model has an $S U(3) \times S U(2)$ gauge symmetry and a $U_{Y}(1) \times U_{R}(1)$ anomaly free global symmetry. As above, the low energy phase that minimizes the number of degrees of freedom is the one that breaks supersymmetry along with both of the global symmetries. The massless spectrum is the same as in the parent chiral $S U(5)$ case. In the $(3-2)$ model, however, the low energy spectrum has been 
computed [16] in a self-consistent weak-Yukawa coupling approximation, where it was noted that the $U_{R}(1)$ breaks along with supersymmetry, leaving intact the $U_{Y}(1)$. The spectrum consists of two massless fermions (a Goldstino and the fermion associated with the unbroken $\left.U_{Y}(1)\right)$ and the $U_{R}(1)$ Goldstone boson. If this is indeed the ground state, then the number of infrared degrees of freedom is not minimized in this weak coupling case.

\section{CONCLUSIONS}

We have considered the low energy structure of two chiral gauge theories, the BarsYankielowicz (BY) model and the generalized Georgi-Glashow (GGG) model. Each contains a core of fermions in complex representation of the gauge group, along with a set of $p$ additional fundamental-anti-fundamental pairs. In each case, for $p$ near but not above the value for which asymptotic freedom is lost, the model will have a weak infrared fixed point and exist in the non-abelian Coulomb phase.

As $p$ drops, the infrared coupling strengthens and one or more phase transitions to strongly coupled phases are expected. Several possible phases have been identified that are consistent with global anomaly matching, and that satisfy the inequality Eq. (1.3) for low enough $p$. One is confinement with the gauge symmetry and additional global symmetries unbroken. Another is confinement with the global symmetry broken to that of the $p=0$ theory. Still another is a Higgs phase, with both gauge and chiral symmetries broken. Both symmetry breaking phases correspond to bilinear condensate formation. The infrared degree of freedom count $f_{I R}$ for each of these phases is shown in Figs. 目 and 2, along with the corresponding ultraviolet count $f_{U V}$.

We have suggested that at each value of $p$, these theories will choose the phase that minimizes the degree of freedom count as defined by $f_{I R}$, or equivalently the phase that minimizes the entropy near freeze-out $\left(S(T) \approx\left(2 \pi^{2} / 45\right) T^{3} f_{I R}\right)$. As may be seen from Figs. 1 and 2, this idea leads to the following picture. As $p$ drops below some critical value, the $p$ fundamental-anti-fundamental pairs condense at some scale $\Lambda$, breaking the full global symmetry to the symmetry of the $p=0$ theory and producing the associated Goldstone bosons. For the remaining theory with fermions in only complex representations, the phase with the global symmetry unbroken and the global anomalies matched by massless fermions is preferred to phases with further global symmetry breaking via bilinear condensates. We have not yet shown that this is true relative to all bilinear condensate formation. Also, this

does not exclude the possibility that some strongly coupled infrared phase (such as a strong non abelian coulomb phase) leads to the smallest value for $f_{I R}$ and is still consistent with 
global anomaly matching.

We extended our discussion to include general condensate formation for one simple example, the $S U(5)$ Georgi-Glashow model with fermions in only complex representations and a single $U(1)$ global symmetry. This symmetry can be broken via only a higher dimensional condensate. For this model, interestingly, we noted that the breaking of the $U(1)$ with confinement and unbroken gauge symmetry leads to the minimum value of $f_{I R}$ among phases that are infrared free. This highlights the important question of the pattern of symmetry breaking in general chiral theories (or any theories for that matter) when arbitrary condensate formation is considered. Higher dimensional condensates could play an important role in the dynamical breaking of symmetries in extensions of the standard model [10]. The enumeration of degrees of freedom in the effective infrared theory is a potentially useful guide to discriminate among the possibilities.

Finally, we commented on two supersymmetric chiral models: the supersymmetric version of the $S U(5)$ Georgi-Glashow model and the closely related $(3-2)$ model. Both have a $U_{R}(1) \times U_{Y}(1)$ global symmetry. In each case, the phase that minimizes the number of massless degrees of freedom corresponds to the breaking of SUSY and both of its global symmetries. In the case of the $(3-2)$ model, however, an analysis in the case of a weak Yukawa coupling (see [3] for a discussion and relevant references) leads to the conclusion that the $U_{Y}(1)$ is not broken. If this truly represents the ground state in the case of weak coupling, then the degree of freedom count is not the minimum among possible phases that respect global anomaly matching.

To summarize, for the nonsupersymmetric chiral gauge theories discussed here, we have identified a variety of possible zero-temperature phases and conjectured that the theories will choose from among them the one that minimizes the infrared degree of freedom count. Whether this can be proven and whether the idea plays a role in a wider class of theories remains to be seen.

\section{Acknowledgments}

We thank Andrew Cohen, Erich Poppitz, Nicholas Read and Robert Shrock for helpful discussions. The work of T.A., Z.D. and F.S. has been partially supported by the US DOE under contract DE-FG-02-92ER-40704. 
[1] R. Ball, Phys. Rept. 182 (1989) 1 and references therein.

[2] S. Raby, S. Dimopoulos and L. Susskind, Nucl. Phys. B169 (1980) 373.

[3] E. Poppitz and S. Trivedi, Annu. Rev. Nucl. Part. Sci 48 (1998) 307. See also references therein.

[4] G. 't Hooft, Recent Developments in Gauge Theories, eds., G. t' Hooft (Plenum Press, New York, 1980).

[5] M. Peskin, Les Houches, Session XXXIX, 1982 - Recent Advances in Field Theory and Statistical Mechanics pag. 217, eds., J. Zuber and R. Stora (North-Holland, Amsterdam, Netherlands 1984).

[6] Non-perturbative Aspects of Chiral Gauge Theories, eds., L. Maiani, G. Rossi and M. Testa, Nucl. Phys. B (Proc. Suppl. ) 29B,C (1992).

[7] T. Appelquist, A. Cohen and M. Schmaltz, Phys. Rev. D60 (1999) 045003.

[8] T. Appelquist, A. Cohen, M. Schmaltz and R. Shrock, Phys. Lett. B459 (1999) 235.

[9] I. Bars and S. Yankielowicz, Phys. Lett. 101B(1981) 159.

[10] F.S. Roux, T. Torma and B. Holdom, Report Numbers: UTPT-99-14, hep-ph/9907540. J. Stern, Report Numbers: IPNO/TH 97-30, hep-ph/9712438; IPNO/TH 97-41, hep$\mathrm{ph} / 9801282$.

[11] E. Eichten, R. Peccei, J. Preskill and D. Zepperenfeld, Nucl. Phys. B268 (1986) 161.

[12] S. Khlebnikov and R. Peccei, Phys. Rev. D48 (1993) 361.

[13] S. Dimopoulos and L. Susskind, Nucl. Phys. B173 (1980) 208.

[14] I. Affleck, M. Dine and N. Seiberg, Phys. Lett. 137B, (1984), 187.

[15] Y. Meurice and G. Veneziano, Phys. Lett. B 141, 69, (1984).

[16] J. Bagger, E. Poppitz and L. Randall, Nucl. Phys. B426 (1994), 3. 